\title{
A Comparison Of Four Series of Cisco NeTWORK PROCESSORS
}

\author{
Sadaf Abaei Senejani ${ }^{1}$, Hossein Karimi ${ }^{2}$ and Javad Rahnama ${ }^{3}$ \\ ${ }^{1}$ Department of Computer Engineering, \\ Pooyesh University, Qom, Iran \\ ${ }^{2}$ Sama Technical and Vocational Training College, \\ Islamic Azad University, Yasouj Branch, Yasouj, Iran \\ ${ }^{3}$ Department of Computer Science and Engineering, \\ Sharif University of Technology, Tehran, Iran.
}

\begin{abstract}
Network processors have created new opportunities by performing more complex calculations. Routers perform the most useful and difficult processing operations. In this paper the routers of VXR 7200, ISR 4451-X, SBC 7600, 7606 have been investigated which their main positive points include scalability, flexibility, providing integrated services, high security, supporting and updating with the lowest cost, and supporting standard protocols of network. In addition, in the current study these routers have been explored from hardware and processor capacity viewpoints separately.
\end{abstract}

\section{KEYWORDS}

Network processors, Network on Chip, parallel computing

\section{INTRODUCTION}

The expansion of science and different application fields which require complex and time taking calculations and in consequence the demand for fastest and more powerful computers have made the need for better structures and new methods more significant. This need can be met through solutions called "paralleled massive computers" or another solution called "clusters of computers". It should be mentioned that both solutions depend highly on intercommunication networks with high efficiency and appropriate operational capacity. Therefore, as a solution combining numerous cores on a single chip via using Network on chip technology for the simplification of communications can be done.

The processors of network are analysed in different parts like Intended data rate and applications, Architecture, Interface, Programmability, Implementation, Cost, and Design wins. But a practical comparison has not been done among operational equipment of network which uses these processors. This comparison can be done between one switch from Intel Company and a similar counterpart in Cisco Company. In the present study the network processors of Cisco Company will be investigated. Cisco was established in 1984 by a group of computer scientists in Stanford University. Cisco system is a leader in network for internet. The solutions of Cisco system are based on internet protocol (IP) which is the basis of internet, developing the activities of companies, education and training departments, and governmental networks in the world. In addition, Cisco provides the most extensive solutions for data transfer, and voice and video in buildings in all universities around the world. Moreover, the solutions of Cisco provide the possibility of the most efficient performance, security, flexibility, and reliability for private and governmental networks. [5]

DOI:10.5121/acii.2016.3402 
Network processor has three different with the old microprocessors [11]:

1- Instruction set in many network processors is based on RISC instruction set.

2- Network Processor instruction set includes special instructions for manipulating bits, calculating the CRC, and search operations.

3- In network processor functions some hardware blocks special accelerate packet processing.

A network processor (that is harvested from its instruction set architecture), is equally important to know which level of the network is in the network processor (the same protocol stack); the levels that can be used in network processors are, are as follows [8]:

- Kernel level, which contains components for high-speed production and transmission of large amounts of data. The nodes are connected upward to kernel-level implementation of basic services such as routing, switching and access control.

- The edge of the network, which forms the core input and output. Core services are complex and are implemented in higher-speed interfaces, services at this point, including routing, switching, network flow, and access control features quality service.

- The access network, which covers all areas of internet delivery. The end user via campus networks, broadband connections and telephone lines becomes available. At this level, there are different protocols and technology together in a relatively low speed.

Network processors can be used both in speed and technique against different package management, which are called level and control level. Level data is performed simple tasks, and more rapid route packets over the network processor spend little time on network, although management procedures are complex. [6]

\section{2- InVESTigation Of Four Series Of Cisco Routers}

The engineers of Cisco work on making advanced products and critical technologies of network and internet like advanced router and switching, voice and image on IP, wireless optical networks, storing networks, security, band width, and content networks. Cisco not only is an inventor in technology and leadership of products, it is also regarded a creator regarding the way of doing businesses. Therefore, this company is a pioneer in using internet and network for supporting customer, selling products, training, recommendations, and financial issues management.

In the following the four series of cisco routers will be explored in separate sections and all the characteristics of each one will be investigated.

\subsection{SBC CISCO7600 SERIES [7][2]}

SBC Cisco7600 series or session border controller (SBC) has been provided based on constant performance of system and scalability of multimedia and also considering the pioneer market of the routers of SBC Cisco7600 series. Through combining SBC with layers 2 and 3 in SBC 
Cisco7600 series, the need for covering networks and independent tools is met and the following features will be provided:

- Protocol and interaction media

- Routing session

- Hosing NAT and firewall survey

- Security and AAA

- Connecting and optimizing inside and between specific virtual network

- Transcoding media with external media server

SBCs do the task of controlling and managing real time of traffic among IP network borders, signalling, data, voice, and video traffic. As part of these functions, SBC is responsible for performing required mother IP connection functions for real time communications like accessing control, Firewall traversal, band with police, accounting, interaction signalling, legal travelling, and the quality of management services. Security solutions of Cisco consist of the quality of service and secure virtualizing capacities for facilitating the support of network operations including voice on IP (VoIP), imaging services, etc.

Voip works on phone system. Telephone network is concerned analog sound data (speech). Phone signal frequency range is almost from 0 to maximum $4 \mathrm{kHz}$. In many cases, for voice transmission,changing the analog signal into digital data help us to make lower cost network. To convert analog signals to digital phone, according to the Nyquist theorem, at least eight Khz frequency is required and also according to the standard of 8-bit samples per period, to transmit the digital broadband, the $64 \mathrm{Kbps}$ analog telephone line is needed. This broadband digital telephone transmission is ideally foundation. But the allocation of $64 \mathrm{Kbps}$ to transfer each audio channel requires costly, especially when it is desired network the size of Earth. With advances in technology, especially in the computer field, new solutions to reduce communication costs were raised that some of these solutions leads to lower bandwidth requirements on telephone transfer. On the VOIP also different standards for converting sound into digital data have been used that they need to $64 \mathrm{Kbps}$ to $8.3 \mathrm{Kbps}$ bandwidth.

Using connector in Cisco 7600 Integrated Switching, not only makes the designing of SBC scalable for VoIP sessions but also it is appropriate for supporting high band width of video sessions like telepresence. Moreover, it is suitable for Service provider to service provider and Service provider to application Service provider. SBC Cisco7600 has unified signalling position and distributed architecture. We draw a SBE Sisco 7600 plan in figure 1.

Supporting industry standard protocol and connector for VoIP and video like session initiation protocol (SIP) and H.323 are also put in it. In addition, SBC Cisco7600 make possible transmission support, convergence services of internet for advanced connector networks (TISPAN IT) which is in H.248 protocol based on performing the distribution of SBC signalling and media current. 


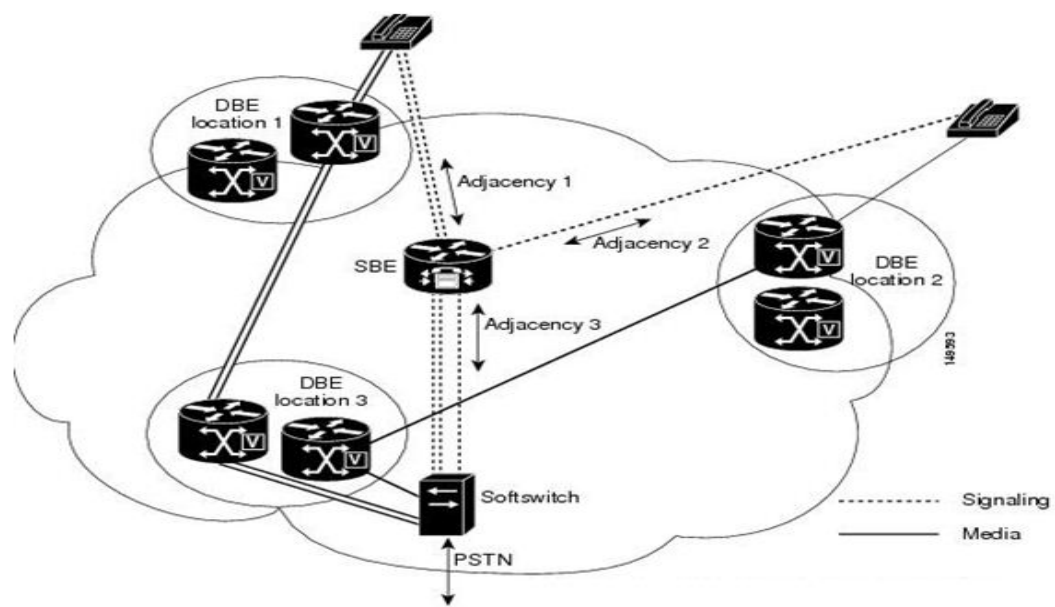

Figure 1. In this diagram adjacencies $1,2,3$ have been associated with the respective DBE

locatiopns. the first (double line) call comes in over adjancency 1 and is routed over adjacency 3. the second (single line) call comes over adjancency 2 and is routed over adjancency 3 . the SBE pickes a DBE from the appropriate location to process the call media.

\subsection{VXR CISCO7200 SERIES [10] [1]}

VXR Cisco7200 series provides significant adaptability and is suitable for operational programs which need Gigabit Ethernet, OC3/STM-1 and connecting with exceptional services of IP. The speed of processing reaches one million packets by using three connectors of Gigabit Ethernet. It also has huge gradient of adaptor port options and incomparable amount of complicated IP service capacities. Cisco provides the last generation of integrated services of router like cisco series of 1800, 2800, and 3800 for applying complex services and operational programs even in the smallest offices from distance. Final success and positioning such complex operations depend on installing and running an efficient and scalable system in aggregation points of network. VXR Cisco7200 is suitable for confronting this challenge and providing clear and ideal programs. The main positive point of VXR Cisco7200 is its scalability and flexibility.

VXR Cisco7200 is normally used for WAN applications, voice on IP (VoIP), security, and different operational programs of IP and has been extended because of its noticeable routing. Scalability and cost effective supporting from the investment and maximum return of the investment are properties which enforce any organization to use VXR Cisco7200 series in the business for the enhancement and reinstall of cisco router.

Processor motor of VXR Cisco7200 provides operational power of one Mega packet per second. VXR Cisco7200 can support at least 6 completely modular adaptors. In addition, as the most powerful platform processing unit, VXR Cisco7200 has price-performance ratio and provides configurations with high density of $1 \mathrm{mpps}$ processing in one point and cost effective price to the customers. Through using VPN router coded security of IP (IPsec) and scalability to 280 Megabit per second for headend can be provided. Security capacities in VXR Cisco7200 include supporting a stateful firewall (any firewall that performs stateful packet inspection (SPI) or stateful inspection), prevention from intrusion, access control lists (ACL), and IPsec. Low price of each input point port in VXR Cisco7200 makes it valuable and let customers to perform enhancement and reinstall of their equipment in a network with high acceptability level. Moreover, processor based on system architecture in these series of products helps to assure that new functions can be used fast and easily through updating simple software. The potentialities of using VXR Cisco7200 include: (as shown in figure 2) 
- Multiprotocol routing of IPv4, IPv6, Internetwork Packet Exchange (IPX), DECnet, Systems Network Architecture (SNA), etc.

- Combining IBM SNA with IP of network

- Security-firewall of stateful. Intrusion Prevention System (IPS), hardware coding for IPSec, Network Address Translation (NAT) and confirming identities, options and accounting (AAA)

- Network Based Application Recognition (NBAR)

- Supporting data, voice, video and integration

- Quality of services (QOS)

- Reflecting the route

- Multiprotocol Label Switching (MPLS)- provider of functions, virtual private networks of (MPLS V PN MPLS, layer 2 and 3), QOS in MPLS, and traffic engineering of MPLS (MPLS-TE)

- Multicast

- Mutilpoint dynamic VPNs (DMV PNs)

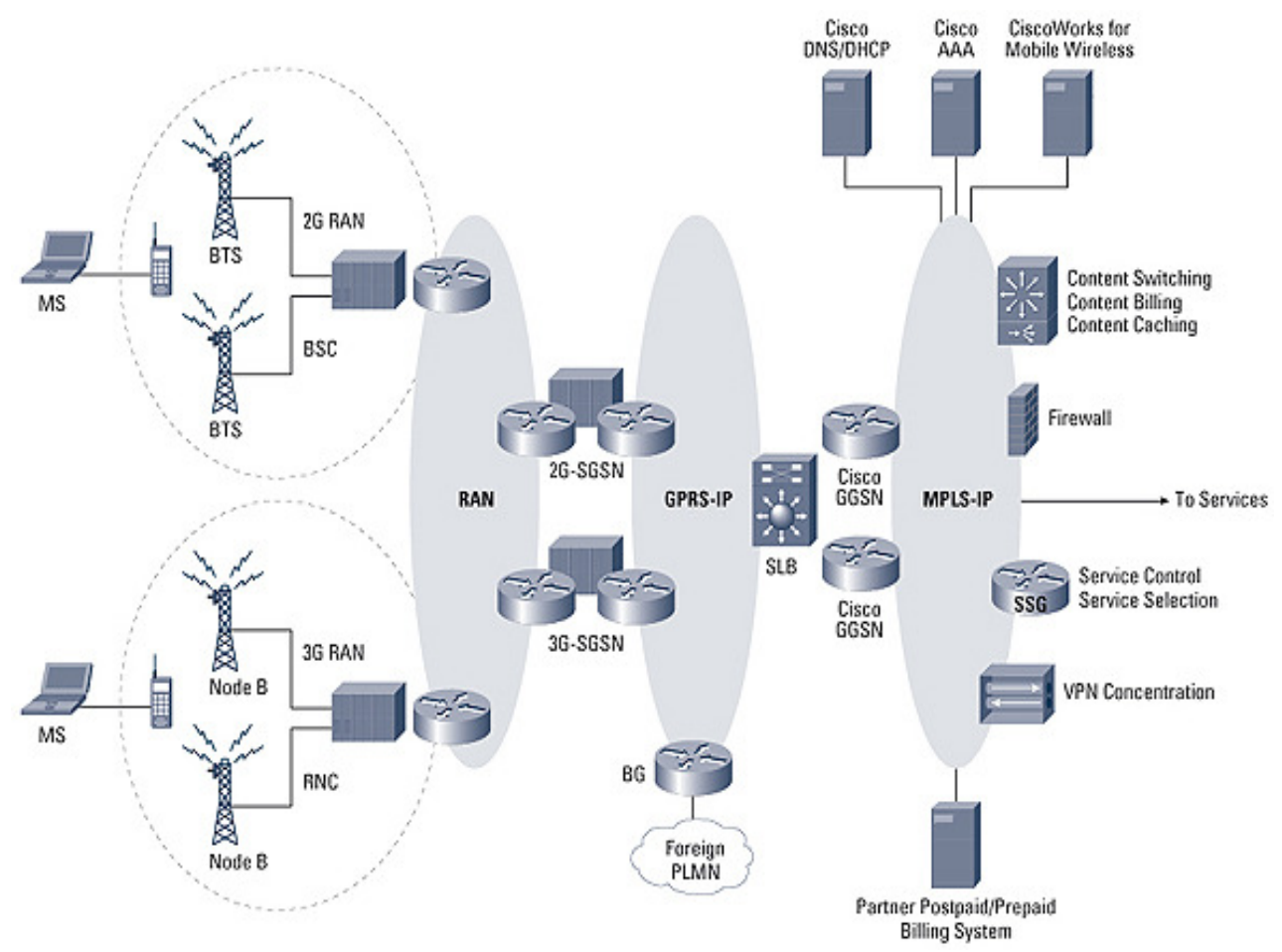

Figure 2. VXR Cisco7200 series Architecture

\subsection{CISCO ISR 4451-X SERIES [4][3][9]}

Cisco ISR 4451-X series has an attempt to create a converged infrastructure for a virtual server, storage and network capacity instead of allocating a series of resources to an special function of services. In comparison to traditional and old routers and substructure switching, ISR cisco platform has achieved $40 \%$ reduction in operation costs. Cisco ISR 4451-Xseries is a converged substructure of a platform for creativity which provides global coordination of network, computation and storing for unprecedented and powerful performance as ALL-IN-ONE with extraordinary low total costs of ownership (TCO). Cisco ISR 4451-X series consists of the 
solution of integrated services routers (ISR), unified computational system of cisco (UCS cisco) and switch 24 module of cisco port. These components together have 2 gigabit calculation power, 6 cores processors, 16 gigabit RAM memory and 2 Tribute local storing capacity. They can also support switching more powerful than Ethernet $(+\mathrm{PoE})$ to achieve advanced industry goals in cisco. Development process of ISR routers from $50 \mathrm{Mbps}$ to $2 \mathrm{Gbps}$ has been shown in figure 3.

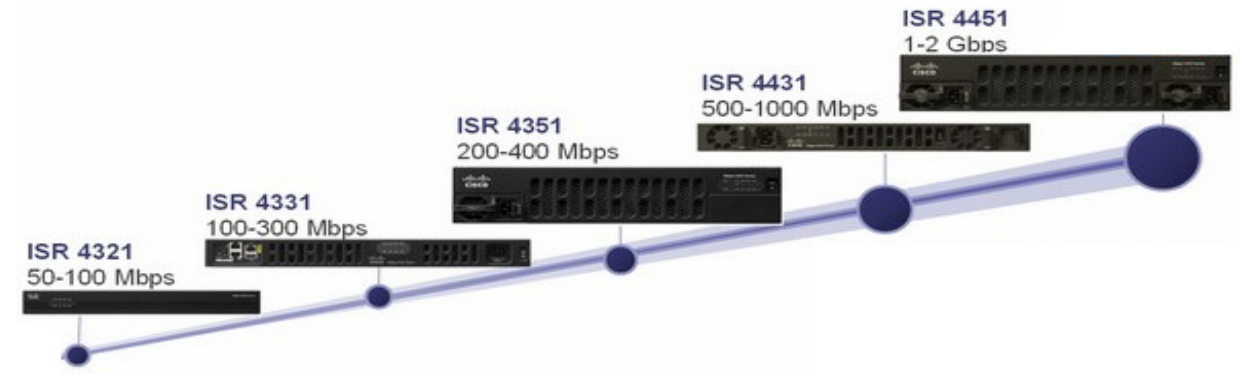

Figure 3. ISR routers development

This platform has provided noticeable advance in router global architecture with highest commercial and security dimensions. This model of platform has included the growth and progressing needs of customers and makes scalability possible in future. In addition, in the future integrated framework of rapid virtual services is put in cisco for more creativity. The solution of converged cisco $4451-\mathrm{X}$ is the biggest advance in routing, security, wireless, and calculations in all-in-one box which can gives complete services like the following:

- Advanced routing

- Integrated switching with $+\mathrm{PoE}$

- Secure VPN

- View and control application (optional)

- Controlling the route of WAN (optional)

- Optimizing WAN (optional)

- Control wireless networks (WLAN) (optional)

- computing the extension of environment through supporting multi hypervisor

Cisco 4451-X provides ideal virtual integrated environment for performing network services in router without any extra hardware. When extra calculation power is needed, cisco UCS E-series adds modular servers to the system. This architecture creates the best method for providing application programs for giving services to users.

\subsection{CISCO 7606 SERIES [12]}

Cisco 7606 is a router with high performance in an operating system of 6 memories in the edge of network. In this cisco multiprotocol switching label has been designed for considering the needs of companies and service providers. These service providers enable the Ethernet carrier to establish substructures of advanced networks through supporting extensive gradient of IP video and media player (voice, video, and data) of system programs in both local service markets and businesses. Through a powerful combination of speed and service in a compact agent, Cisco 7606 is a significant option for various application programs. In the present point of organizational edge and city network edge (POP), Cisco 7606 series are new standard sets as part of progressing industry of cisco router. Cisco 7606 with transfer rate of 240 gigabit per second distribution and total capacitance of 480 gigabit per second provides high performance and reliability for the processor and feeding resource. Cisco 7606 is flexible and ideal for dealing with application 
programs with high efficiency. Cisco 7606 has high option capacity for supporting various application programs. In table 1 a comparison of mentioned series in this paper has been summarized.

Table 1: a comparison of cisco series (VXR 7200, SBC7600, ISR 445-X, 7606)

\begin{tabular}{|c|c|c|c|c|}
\hline feature & VXR7200 & SBC7600 & ISR 445-X & 7606 \\
\hline $\begin{array}{l}\text { Supporting } \\
\text { services }\end{array}$ & $\begin{array}{l}\text { Cost effective } \\
\text { supporting }\end{array}$ & Active & Yes & Yes \\
\hline scalability & Yes & Yes & Yes & Yes \\
\hline flexibility & Yes & Yes & Yes & Yes \\
\hline business & Yes & Yes & Yes & Yes \\
\hline security & $\begin{array}{l}\text { Supporting stateful, } \\
\text { prevention }\end{array}$ & $\begin{array}{l}\text { Supporting IPsec } \\
\text { and security }\end{array}$ & $\begin{array}{l}\text { Recognizing } \\
\text { identity, providing } \\
\text { MACsec }\end{array}$ & $\begin{array}{l}\text { Module-IP } \\
\text { (IPsec), } \\
\text { analysis and } \\
\text { identifying } \\
\text { system of } \\
\text { network }\end{array}$ \\
\hline $\begin{array}{l}\text { Supporting } \\
\text { protocol }\end{array}$ & IPV4,IPV6,... & $\begin{array}{l}\text { initiation session } \\
\text { protocol and } \\
\text { H.323, H.248 }\end{array}$ & & \\
\hline VPN & $\begin{array}{l}\text { Using VPN } \\
\text { (+VAM2) }\end{array}$ & $\begin{array}{l}\text { Via layer } 3 \text { of } \\
\text { network }\end{array}$ & Secure VPN & \\
\hline $\begin{array}{l}\text { Processing } \\
\text { power }\end{array}$ & $\begin{array}{l}\text { Operational power } \\
\text { of } 1 \mathrm{Mpps}\end{array}$ & $\begin{array}{l}\text { Using control } \\
\text { module }\end{array}$ & $\begin{array}{l}6 \text { cores processors, } \\
16 \text { gigabyte } \\
\text { memory }\end{array}$ & $\begin{array}{l}\text { Total power of } \\
480 \text { gigabit per } \\
\text { second }\end{array}$ \\
\hline $\begin{array}{l}\text { Processing } \\
\text { speed }\end{array}$ & $\begin{array}{l}\text { One million packet } \\
\text { in three ports of } \\
\text { Ethernet gigabit }\end{array}$ & $\begin{array}{l}10 \text { gigabit in each } \\
\text { slot of processing } \\
\text { capacity }\end{array}$ & $\begin{array}{l}\text { Switch module of } \\
24 \text { port, up to } 2 \\
\text { gigabit } \\
\text { performance }\end{array}$ & $\begin{array}{l}\text { Transfer rate } \\
\text { up to } 24 \\
\text { gigabit per } \\
\text { second }\end{array}$ \\
\hline $\begin{array}{l}\text { Integrated } \\
\text { services }\end{array}$ & The last generation & Yes & $\begin{array}{l}\text { Rapid integration } \\
\text { services }\end{array}$ & Yes \\
\hline QOS & Yes & Yes & Yes & \\
\hline Routing & $\begin{array}{l}\text { Multiprotocol } \\
\text { routings }\end{array}$ & $\begin{array}{l}\text { Routing based on } \\
\text { the least cost }\end{array}$ & advanced & $\begin{array}{l}\text { The capacity } \\
\text { of routing, } \\
\text { protection of } \\
\text { processor }\end{array}$ \\
\hline
\end{tabular}

\section{FUTURE WORKS}

In the future expansion of the Internet of Things, CPU usage also will be greater. Fast connections with the lowest latency and the highest rate of packet sending is the perfect condition for each network. Given that in 2020, Moore's Law will come to the end, so it will be very difficult to make changes to the chip later on. Although methods such as Super pipeline and super scalar and like that, partly improve the quality of processors in that time. However, due to finishing increase of processor speed, alternative is use of network processors which has more cost than a fast processor. Use of distributed network processors can lead to new ways of fast connections. Also discovering new routing network algorithms can lead to processing with high speed in the future. 


\section{CONCLuSion}

Network processors are special types of central processing of CPU which are used for network equipment like routers. They are used for the production of different kinds of network equipment like network switching, and identifying intrusion system. In this study network processors which are used in cisco network products have been analysed and compared. Network on a chip (NOC) is a communication subsystem inside an integrated circuit which connects IP cores in a system on a chip in network processors. Technology of NOC of network theory and the ways of on a chip communication has noticeable advances in comparison to links based on gateway. These improved architectures cause an increase in the speed of computations after 2020 (the end of Moore's law). Advanced services of cisco are comprehensive series of professional engineering. In this study, features like supporting network solutions, the highest level of access, quality of services, security for a special network and returning business and investment through network with high efficiency in different series of cisco was dealt with.

\section{REFERENCES}

[1] Conlan, P. J. (2009). Cisco Network Professional's Advanced Internetworking Guide (CCNP Series). John Wiley \& Sons.

[2] IPSec, V. P. N. (2005). Catalyst 6500 Series Switches and Cisco 7600 Series Routers with IPSec VPN SPA Module-Security Policy version 1.2.

[3] Headquarters, A. (2014). Cisco Collaboration Systems Release.

[4] Headquarters, A. (2015). Cisco Integrated Services Routers (ISR) 4400 Series Security Target.

[5] Jafarabad, M., Rafie, M., Khodkari, S., \& Alizadeh, Z. (2012). Encryption of Compressed MultiMedia Data. International Journal of Computer Applications, 59(19), 51-55.

[6] Mikurak, M. G. (2003). U.S. Patent No. 6,606,744. Washington, DC: U.S. Patent and Trademark Office.

[7] Mohácsi, J. (2005). IPv6 router configuration. Diunduh tanggal, 20.

[8] Paulin, P. G., Pilkington, C., \& Bensoudane, E. (2002). StepNP: A system-level exploration platform for network processors. IEEE Design \& Test of Computers, 19(6), 17-26.

[9] Radan, M., \& Keslassy, I. (2015, April). Tapping into the router's unutilized processing power. In Computer Communications (INFOCOM), 2015 IEEE Conference on (pp. 2569-2577). IEEE.

[10] Shaikh, F. A., McClellan, S., Singh, M., \& Chakravarthy, S. K. (2002). End-to-end testing of IP QoS mechanisms. Computer, (5), 80-87.

[11] Taylor, M. B., Kim, J., Miller, J., Wentzlaff, D., Ghodrat, F., Greenwald, B., ... \& Ma, A. (2002). The Raw microprocessor: A computational fabric for software circuits and general-purpose programs. IEEE micro, 22(2), 25-35.

[12] Wolchok, S., Wustrow, E., Isabel, D., \& Halderman, J. A. (2012). Attacking the Washington, DC Internet voting system. In Financial Cryptography and Data Security (pp. 114-128). Springer Berlin Heidelberg. 